\title{
A Comparison of Prepulse Inhibition in Pre- and Postmenopausal Women and Age-Matched Men
}

\author{
Veena Kumari*,', Ingrid Aasen', Andrew Papadopoulos ${ }^{2}$, Fanta Bojang', Lucia Poon ${ }^{3}$, Rozmin Halari $^{2}$ and \\ Anthony J Cleare Cl, $^{2,3}$ \\ 'King's College London, Department of Psychology, Institute of Psychiatry, London, UK; ${ }^{2}$ King's College London, Division of Psychological Medicine \\ and Psychiatry, Institute of Psychiatry, London, UK; ${ }^{3}$ Affective Disorder Unit, Bethlem Royal Hospital, Beckenham, Kent, UK
}

\begin{abstract}
Prepulse inhibition (PPI) of the startle response is sensitive to sex with women showing less PPI compared with age-matched men and varies according to the menstrual cycle in women. Relatively less is known about sex differences in prepulse facilitation (PPF). To examine further the roles of sex and circulating sex hormones, pre- $(n=20)$ and postmenopausal women $(n=20)$ were compared with men of similar ages ( $n=17,18-40$ years; $n=18,55-69$ years). All participants were assessed on PPI and PPF, and provided saliva samples for measurement of $17 \beta$-estradiol (estrogen) and testosterone. Premenopausal women showed less PPI compared with age-matched men, with no significant difference in PPF. Postmenopausal women did not differ in PPI but showed more PPF than age-matched men. There was less PPI and PPF in older, relative to young, men; pre- and postmenopausal women did not differ significantly. PPI showed no association with the levels of sex hormones. PPF showed small positive associations with both the levels of estrogen and testosterone, especially in young men. The present findings extend recent observations in mice showing less PPI in premenopausal, but not postmenopausal, female compared with male mice of similar ages (Ison and Allen, Behav Brain Res, 2007) to humans. There appear to be no substantial relationships between individual differences in endogenous levels of sex hormones and PPl; fluctuations within an individual may have a stronger role.

Neuropsychopharmacology (2008) 33, 2610-26 18; doi:10.1038/sj.npp. I 301670; published online 23 January 2008
\end{abstract}

Keywords: acoustic startle; sensorimotor gating; sex difference; estrogen; testosterone

\section{INTRODUCTION}

The startle reflex (SR) consists of a set of reflexive, involuntary responses to a sudden, intense stimulus (Landis and Hunt, 1939). The simple startle reflexive response is known to show several forms of plasticity, including prepulse inhibition (PPI; occurs when the startling stimulus is preceded by a prepulse by $30-500 \mathrm{~ms}$ ) and prepulse facilitation (PPF; occurs when the startling stimulus is preceded by a prepulse by $>500 \mathrm{~ms}$ ) (Hoffman and Searle, 1968; Graham, 1975; Graham and Murray, 1977). PPI is considered to provide an operational index of sensorimotor gating (Braff and Geyer, 1990). PPF has been suggested to reflect sustained attention (Dawson et al, 1997) or sensory enhancement linked with modality-specific selective attention (Anthony, 1985).

\footnotetext{
*Correspondence: Professor $\vee$ Kumari, King's College London, Department of Psychology, P078, Institute of Psychiatry, De Crespigny Park, London SE5 8AF, UK, Tel: +00 44207848 0233, Fax: + 0044 207848 0860, E-mail: v.kumari@iop.kcl.ac.uk

Received I5 October 2007; revised II December 2007; accepted I I December 2007
}

There are sex differences in PPI. In healthy populations, women when tested regardless of where they are in their menstrual cycle show less PPI than men (Swerdlow et al, 1993, 1997, 1999; Abel et al, 1998; Kumari et al, 2003, 2004; Aasen et al, 2005) even when possible confounds such as cigarette smoking are controlled for (Swerdlow et al, 1999). The same phenomenon (ie, less PPI in females than males) has been observed in rats (Koch, 1998; Faraday et al, 1999). Sex differences in PPI are considered to be mediated by fluctuating hormones over the ovarian cycle based on the observations of more PPI in women during the (low estrogen) follicular phase relative to the (high estrogen) luteal phase (Swerdlow et al, 1997; Jovanovic et al, 2004). There is no specific research examining sex differences in PPF. There is some evidence to suggest that women might display higher PPF than men at shorter intervals (1000 and $2000 \mathrm{~ms}$ ) (Kumari et al, 2003; Aasen et al, 2005), leading to the proposition that sex differences in human sensorimotor gating might represent a general downshift in the inhibition curve and upward shift in the facilitation curve in women compared with men (Kumari et al, 2003).

The present study examined the roles of sex and circulating sex hormones in sexual dimorphism found in sensorimotor gating by examining PPI and PPF in pre- and 
postmenopausal women compared with age-matched men and taking relevant hormonal measurements. Menopause in women occurs around the age of 52 years, and is marked by the cessation of ovulation and a dramatic decline in the production of the ovarian hormone estrogen (Halbreich, 1995). Very recently, Ison and Allen (2007) have shown that pre- but not postmenopausal female CBA/CaJ mice show less PPI than male mice of similar age, supporting further that neural mechanisms underlying PPI are sensitive to sex hormones. We hypothesized that (i) premenopausal women would show less PPI compared with young men of similar ages, (ii) postmenopausal women and similarly aged men would show no or a reduced sex difference in PPI, and (iii) PPI would be negatively correlated with estrogen level especially in premenopausal women. An opposite pattern of effects was expected for PPF but with limited confidence, given the lack of robust previous data on that part of our investigation.

\section{MATERIALS AND METHODS}

\section{Subjects and Design}

The study employed a cross-sectional $2 \times 2$ design, involving four groups: group 1, regularly menstruating women (aged 18-40 years); group 2, young men (aged 18-40 years); group 3, postmenopausal women (aged 55-69 years); and group 4, older men (aged 55-69 years).

Eighty volunteers ( $n=20$ /group) were initially recruited to take part. Of these, startle data in five male participants were unusable. Two participants were nonresponders (average amplitude $<25$ units over the first four pulsealone trials), two had a low response rate $(<70 \%$ response probability), and one participant was excluded because of noisy data (unstable baseline). The final sample thus consisted of 75 participants: 20 premenopausal women (mean age $\pm 1 \mathrm{SD}=24.95 \pm 5.06$ years), 17 young men $(28.12 \pm$ 5.23 years), 20 postmenopausal women ( $59.75 \pm 3.43$ years), and 18 aged men $(62.33 \pm 4.28$ years $)$.

Exclusion criteria for all participants included (i) any ear disorder, (ii) left-handedness, (iii) first-degree relative with a history of mental illness, and (iv) diagnosis of a psychiatric or neurological disorder or a current or past primary diagnosis of substance misuse. All potential participants underwent a semistructured medical screening for a history of mental illness, alcohol dependency, and drug abuse (also confirmed by urine analysis in a random subset), and were screened for intact auditory abilities using an audiometer (Kamplex, AS7) at $40 \mathrm{~dB}$ (A) $(1000 \mathrm{~Hz})$ before being accepted as study subjects. In addition, the premenopausal women were required to have 26-30 days menstrual cycle determined using the standard 'counting forward and backward' method.

Postmenopause was defined as 12 consecutive months of amenorrhea (Park et al, 2002). None of the premenopausal women used any oral contraceptives, and none of the postmenopausal women used any hormone replacement. No young participant was on any regular medication. Eight (four men and four women) of 38 older participants were on blood pressure- and/or cholesterol-lowering medication. Three men (two young and one aged) and two women (both postmenopausal) were light smokers (5-12 cigarettes/day).
The study was approved by the Local Research Ethics Committee. All participants provided written informed consent.

\section{Prepulse Inhibition and Facilitation of the Startle Response}

Startle response measurement. A commercial computerized human startle response monitoring system (Mark II; SR Lab, San Diego, CA) was used for the delivery of the acoustic startle stimuli and for the recording/scoring of the electromyographic (EMG) activity for $250 \mathrm{~ms}$ starting from the onset of the stimulus. Stimuli were presented to participants binaurally through headphones (Telephonics, TDH 39P) while they were sitting in a moderately lit soundproof laboratory. The eyeblink component of the startle response was indexed by recording EMG activity of the orbicularis oculi muscle by positioning two miniature silver/silver chloride electrodes filled with Dracard electrolyte paste (SLE, Croydon) directly beneath the right eye. The ground electrode was attached to the mastoid behind the right ear. The EMG signal amplification gain control was kept constant for all participants, and recorded EMG activity was band-pass filtered, as recommended by the SR Lab. A $50-\mathrm{Hz}$ filter was used to eliminate the $50-\mathrm{Hz}$ interference. EMG data were scored off-line by the analytic program of this system for response amplitude (analogue-to-digit units; 1 unit $=2.62 \mu \mathrm{V}$ ) and latency to response peak (in $\mathrm{ms}$ ). Latency to response onset was defined by a shift of 20 digital units from the baseline value occurring within $18-100 \mathrm{~ms}$ after the stimulus. The latency to response peak was determined as the point of maximal amplitude that occurred within $120 \mathrm{~ms}$ from the acoustic stimulus. If the onset and peak latencies differed by more than $95 \mathrm{~ms}$ or the baseline values shifted by more than 50 units, then the responses were rejected ( $<5 \%$ trials in total).

Experimental paradigm and procedure. The pulse-alone stimulus was a $40-\mathrm{ms}$ presentation of $115-\mathrm{dB}$ (A) white noise and the prepulse stimulus was a $20-\mathrm{ms}$ presentation of 84-dB (A) noise; both presented over 70-dB (A) continuous background noise. The session began with a 5-min acclimatization period consisting of $70-\mathrm{dB}$ (A) continuous white noise. Participants received 100 startle stimuli in all. Ninety-nine trials, in three blocks of 33 trials each, followed the initial pulse-alone trial. There was a range of prepulseto-pulse intervals (prepulse onset to pulse onset) to elicit PPI (30,60, 120, 240, and $480 \mathrm{~ms}$ ) and PPF (1000, 2000, 3000,4500 , and $6000 \mathrm{~ms}$ ). Each block consisted of three pulse-alone trials and three trials with each prepulse-topulse interval presented to participants in a pseudorandom order with a mean intertrial interval of $15 \mathrm{~s}$ (range: 9-23 s). The session lasted about $30 \mathrm{~min}$. Participants were not given any specific instructions to either attend to or ignore the auditory stimuli. They were instructed to keep their eyes open during the experiment.

Eleven of 20 premenopausal young women were tested during the first half, and the remaining 9 during the second half, of their menstrual cycle determined on the basis of the first day of their last menstrual period and the average length of their last three menstrual cycles. The smoking participants (five in total) were requested not to smoke a cigarette for at least $1 \mathrm{~h}$ prior to testing. 


\section{Saliva Sampling}

To measure $17 \beta$-estradiol (chief estrogen) and testosterone levels, one saliva sample was collected just before the startle experiment and three additional samples were taken at 30 -min intervals throughout the testing session. Participants were asked not to eat, drink, chew gum, or brush their teeth $30 \mathrm{~min}$ before sampling. Otherwise, they had to rinse their mouth thoroughly with cold water 5 min prior to sample collection. Samples were not collected when oral diseases, inflammation, or lesions existed (blood contamination). The participants passed saliva (minimum of $2 \mathrm{ml}$ ) through a plastic straw into a 3-ml polypropylene 'Cryovial' (Thermoscientific, Birmingham, UK). Following the manufacturer's recommendation, the samples were frozen at $-20^{\circ} \mathrm{C}$ and stored. Prior to analysis, saliva specimens collected at each time point were defrosted, mixed, and, after centrifugation at 3500 r.p.m. at room temperature (RT), equal volumes were taken from each specimen to form a pooled specimen. Small aliquots $(50 \mu \mathrm{l})$ of these pooled specimens were separately analyzed (by AP) in duplicate, for estradiol and testosterone, by Luminescence immunoassay (IBL, Hamburg, Germany). If the difference between the duplicate measurements was more than $10 \%$, then the analysis was repeated. Briefly, using the Genesis 100 Robotic Sample Processor (Tecan UK, Theale, Reading, UK), $50 \mu$ of the test saliva or standard was added to the well of microtitration strips. This was followed by $50 \mu \mathrm{l}$ of a solution of the enzyme-labeled hormone and $50 \mu \mathrm{l}$ of the hormone antibody. After an incubation of $4 \mathrm{~h}$ at RT, the incubation solution was discarded and the wells were washed four times with $250 \mu$ l of wash buffer and $50 \mu \mathrm{l}$ of chemiluminescence reagent added. The luminescence of the bound fraction was measured in a Berthold luminometer (MPL1, Berthold Detection Systems, Pforzheim, Germany), which was linked to MikroWin 2000 Version 4 (Microtek Laborsysteme, Ovoroth, Germany) for immunoassay data processing. Hormone concentrations were read off a calibration graph, constructed from a series of hormone standards. The day-to-day performance of the assays was monitored using each kit's saliva control specimens and also commercial control sera (Immunoassay Plus; Bio-Rad, Hemel Hempstead, Herts, UK), which had been suitably diluted with each kit's zero standard. For $17 \beta$-estradiol, the range of standards was from 0 to $64 \mathrm{pg} / \mathrm{ml}$ : the analytical sensitivity was $0.3 \mathrm{pg} / \mathrm{ml}$; intra-assay precision $(\mathrm{CV})<10 \%$ at $10-40 \mathrm{pg} / \mathrm{ml}$; and interassay precision $c a .12 \%$ at $33 \mathrm{pg} / \mathrm{ml}$. For testosterone, the range of standards was from 0 to $760 \mathrm{pg} / \mathrm{ml}$ : the analytical sensitivity was $1.8 \mathrm{pg} / \mathrm{ml}$; and inter- and intra-assay precision $(C V)<10 \%$ at $20-540 \mathrm{pg} / \mathrm{ml}$ (for further details see http://www.ibl-hamburg.com/index,1.0.html; reference numbers RE62031 (testosterone) and RE62041 (17 $\beta$-estradiol)).

\section{Statistical Analysis}

All analyses were carried out using Statistical Package for the Social Sciences (SPSS, version 15) with $\alpha$-level for significance testing maintained at $p \leqslant 0.05$ unless otherwise specified.

\section{Startle Measures}

Startle modulation was computed as percentage reduction of the amplitude over pulse-alone trials; PPI and
$\mathrm{PPF}=(a-b) / a \times 100$, where ' $a$ ' $=$ amplitude over pulsealone trials and ' $b$ ' $=$ amplitude over prepulse trials. This procedure corrects for the influence of individual differences in startle amplitude (Mansbach et al, 1988). The PPI data were analyzed separately to PPF (since PPF would be expressed as a negative value), an approach used in previous studies investigating both PPI and PPF (eg, Hazlett et al, 1998; Kumari et al, 2004; Aasen et al, 2005).

The effects of sex in the premenopausal/young and postmenopausal/relatively older groups in PPI were evaluated with a $2 \times 2 \times 5$ (sex (women, men) $\times$ age group (aged $18-40$, aged $55-69$ years) $\times$ trial type (inhibition by 30,60 , 120,240 , and $480 \mathrm{~ms}$ prepulse trials)) multivarivate analyses of variance (MANOVA, Wilk's F) with trial type as a withinsubject factor, and sex and age group as the between-subject factors. The effects of sex in PPF were evaluated, separate to PPI, with a $2 \times 2 \times 5$ (sex $\times$ age group $\times$ trial type (facilitation by $1000,2000,3000,4500$, and $6000 \mathrm{~ms}$ trials)) MANOVA with trial type as a within-subject factor, and sex and age group as the between-subject factors. Significant interactions were followed by lower order MANOVAs and post hoc mean comparisons as appropriate. Following observation of a significant main effect of age group in pulse-alone amplitude (see Results), the effects of sex in the premenopausal/young and postmenopausal/ relatively older groups in PPI and PPF were reevaluated with $2 \times 2 \times 5$ ( $\operatorname{sex} \times$ age group $\times$ trial type) MANOVAs in a subsample with comparable mean pulse-alone amplitude in all four groups (achieved by excluding three premenopausal women and three young men with the smallest pulse-alone amplitudes, and four postmenopausal women and four aged men with the largest pulse-alone amplitudes).

The effects of sex and age group in the amplitude and habituation of the startle response over the entire session were evaluated by a $2 \times 2 \times 3 \quad(\operatorname{sex} \times$ age group $\times$ block (three blocks of three pulse-alone trails each)) MANOVA, followed by lower order MANOVAs and post hoc comparisons as appropriate. Following observation of a significant main effect of age group in pulse-alone amplitude (see Results), the relationships between mean pulse-alone amplitude and PPI/PPF over different trial types were explored (using Pearson's $r$ ).

The influence of sex and age group on initial reactivity was examined using a one-way analysis of variance (ANOVA) on amplitude over the first pulse-alone trial.

The latencies to response onset were analyzed by $2 \times 2 \times 6 \quad($ sex $\times$ age group $\times$ trial type (pulse-alone and $\mathrm{PPI} / \mathrm{PPF}$ trials)) MANOVA with trial type as a withinsubject factor, and sex and age group as a between-subject factor, followed by lower order MANOVAs and post hoc mean comparisons as appropriate.

\section{Sex Hormones, and PPI and PPF}

$17 \beta$-Estradiol and testosterone levels were analyzed (separately) using a $2 \times 2$ (sex $\times$ age group) ANOVA. Correlational analyses (Pearson's $r$ ) examined the relationships between sex hormones, and PPI and PPF. To restrict the number of correlations performed, only $120-\mathrm{ms}$ PPI and 4500-ms PPF scores were examined, as these were the most effective intervals. Three series of analyses were conducted. The first series examined the above relationships in the 
entire sample, the second series examined the sample classified by sex and age group, and the third series examined each of the four groups separately. This was done due to the sex and age group effects on levels of certain hormones (see Results).

\section{RESULTS}

\section{Startle Measures}

Prepulse inhibition. The three-way sex $\times$ age group $\times$ trial type MANOVA revealed significant main effects of sex $(\mathrm{F}(1,71)=10.17, p=0.002)$, indicating less PPI in women than men; age group $(\mathrm{F}(1,71)=10.77, p=0.002)$, indicating more PPI in young, relative to older, groups; and of trial type $(\mathrm{F}(4,68)=4.01, p=0.006)$, indicating more PPI on the $120-\mathrm{ms}$ prepulse trials than on the $30-\mathrm{ms}$ and $480-\mathrm{ms}$ prepulse trials (quadratic $\mathrm{F}(1,71)=5.44, p=0.02$ ). Importantly, a significant sex $\times$ age group $\times$ trial type interaction $(\mathrm{F}(4,68)=3.11, p=0.02)$ was also present (see Figure 1).

In young participants, there was a highly significant effect of $\operatorname{sex}(F(1,35)=9.51, p=0.004)$, indicating less PPI in premenopausal women than men aged 18-40 years across all PPI trials; sex $\times$ trial type interaction was not significant $(\mathrm{F}(4,32)=1.52, p=0.22)$ (Figure 1$)$. There was no effect of $\operatorname{sex}(\mathrm{F}(1,36)=2.07, p=0.16)$ or a sex $\times$ trial type interaction $(\mathrm{F}(4,33)=2.07, p=0.11)$ in older (55-69 years) participants.

The effect of age group in PPI was highly significant in men $(\mathrm{F}(1,33)=13.88, p=0.001$; less PPI in older men) and interacted marginally with trial type $(\mathrm{F}(4,30)=2.70$, $p=0.05)$. Although post hoc mean comparison indicated the age group effect in men to be present at all trial types $(p \leqslant 0.05)$, it was most strongly seen for 120 -ms trial type (quadratic $\mathrm{F}(1,33)=9.67, p=0.004)$. The effect of age group was not significant in women $(\mathrm{F}(1,38)=1.90, p=0.18)$; age group $\times$ trial type interaction also failed to reach significance $(\mathrm{F}(4,35)=2.22, p=0.09)$.

Further between-groups analyses showed that premenopausal women and older men did not differ in PPI $(\mathrm{F}(1,36)=$ $0.006, p=0.94$ ) at any trial type (group $\times$ trial type: $\mathrm{F}(4,33)=$ $1.28, p=0.30)$. Young men showed greater PPI than postmenopausal women $(\mathrm{F}(1,35)=15.89, p<0.001)$ across all intervals (group $\times$ trial type: $\mathrm{F}(4,32)=1.29, p=0.29$ ).

Subsample analyses: In the subsample involving four groups with comparable mean pulse-alone amplitude (group: $\mathrm{F}(3,61)=0.61, p=0.61$; mean \pm 1 SEM pulse-alone amplitude (in arbitrary units): 17 premenopausal women $=$ $391.55 \pm 52.92$; 14 young $\operatorname{men}=300.61 \pm 58.31$; 16 postmenopausal women $=342.57 \pm 54.54$; and 14 older men $=$ $393.57 \pm 58.31$ ), the MANOVA revealed significant main effects of sex $(\mathrm{F}(1,57)=9.50, p=0.004)$, age group $(\mathrm{F}(1,57)=9.08, p=0.004)$, and trial type $(\mathrm{F}(4,68)=4.85$, $p=0.002)$; and a significant sex $\times$ age group $\times$ trial type interaction $(\mathrm{F}(4,54)=2.71, p=0.04)$. These effects indicated the same pattern of results as described earlier for the whole sample and displayed in Figure 1 (subsample mean \pm 1 SEM PPI (percentage of change) with $30,60,120,240$, and $480 \mathrm{~ms}$ trials, respectively, in premenopausal women: $11.46 \pm 5.35$, $14.49 \pm 9.03, \quad 15.77 \pm 7.87,6.44 \pm 5.01, \quad 0.91 \pm 4.80 ;$ young

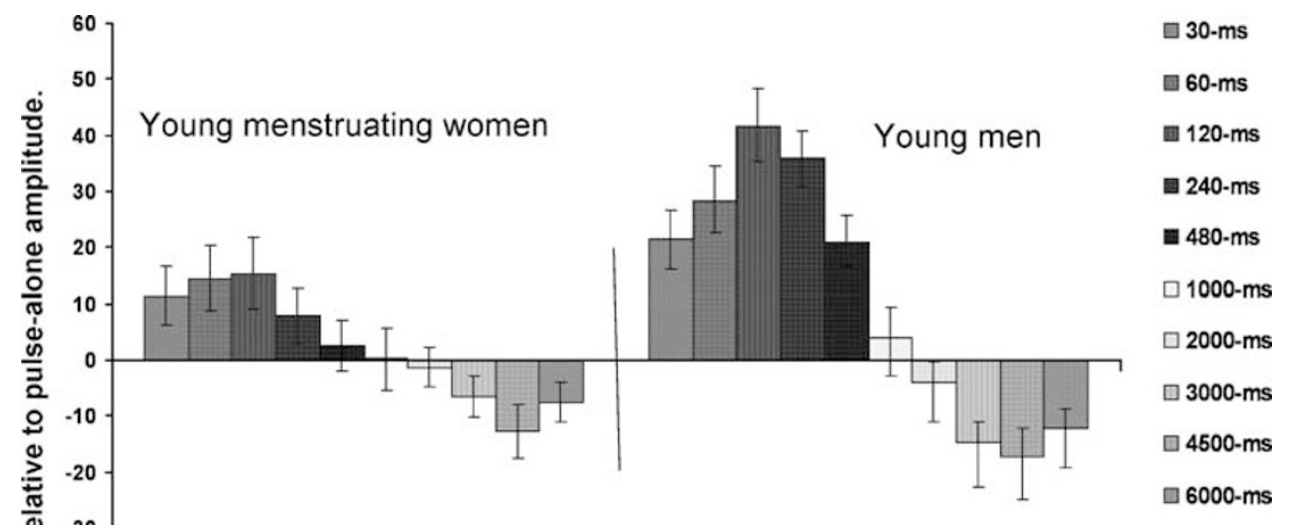

Figure I Mean startle modulation with a range of prepulse-to-pulse intervals in women and men. Error bars display \pm I SEM. 
men: $22.23 \pm 5.90, \quad 28.33 \pm 9.95, \quad 46.23 \pm 8.67, \quad 38.09 \pm 5.52$, and $19.55 \pm 5.29$; postmenopausal women: $-5.80 \pm 5.52$, $-9.63 \pm 9.31,-3.83 \pm 8.11,9.36 \pm 5.17$, and $2.94 \pm 4.95$; older men: $11.15 \pm 5.90,9.39 \pm 9.95,12.55 \pm 8.67,11.15 \pm 5.52$, and $6.65 \pm 5.29)$.

In young participants, there was a significant effect of sex $(\mathrm{F}(1,29)=8.93, p=0.006)$, indicating less PPI in premenopausal women than young men across all PPI trials; sex $\times$ trial type interaction was not significant $(\mathrm{F}(4,26)=$ $1.74, p=0.17)$. There was no effect of $\operatorname{sex}(\mathrm{F}(1,28)=2.13$, $p=0.16)$ or a sex $\times$ trial type interaction $(\mathrm{F}(4,25)=1.61$, $p=0.21$ ) in older participants.

The effect of age group in PPI was highly significant in men $(\mathrm{F}(1,26)=10.09, p=0.003$; less PPI in older men) and interacted with trial type $(\mathrm{F}(4,23)=3.24, p=0.03)$. The effect of age group was not significant in women $(F(1$, $31)=1.88, p=0.18)$.

Further between-group analyses showed that premenopausal women and older men did not differ in PPI $(\mathrm{F}(1,29)=0.003, p=0.95)$. Young men showed greater PPI than postmenopausal women $(\mathrm{F}(1,28)=13.81, p=0.001)$ especially with 30 - to $240-\mathrm{ms}$ prepulse-to-pulse intervals (group $\times$ trial type: $\mathrm{F}(4,25)=2.78, p=0.05)$.

Prepulse facilitation. There was only a significant main effect of trial type $(\mathrm{F}(4,68)=11.26, p<0.001)$ with significant linear $(\mathrm{F}(1,71)=25.02, p<0.001)$, quadratic $(\mathrm{F}(1,71)=$ 8.07, $p=0.006)$, and cubic relationships $(\mathrm{F}(1,71)=4.83$, $p=0.03$ ) (Figure 1). There were trends for the main effect of age group $(\mathrm{F}(1,71)=3.56, p=0.06)$ and a sex $\times$ age group interaction $(\mathrm{F}(1,71)=2.95, p<0.09)$. Sex or age group did not interact with trial type (all $\mathrm{F}<1$ ).

Premenopausal women and young men did not differ in PPF (sex: $F(1,35)=0.25, p=0.62$ ). Postmenopausal women showed greater PPF than older men (aged 55-69 years) $(\mathrm{F}(1,36)=5.65, p=0.02)$; this male group did not show PPF (Figure 1).

Pre- and postmenopausal women showed comparable PPF (age group: $\mathrm{F}(1,38)=0.02, p=0.89$ ) (Figure 1). Reflecting the finding noted above, young men showed greater PPF than older men (who did not show PPF) (age group: $\mathrm{F}(1,33)=5.65, p=0.02)$.

Further between-group analyses revealed that premenopausal women showed greater PPF than older men (group: $\mathrm{F}(1,36)=8.50, p=0.006$ ) at all intervals (group $\times$ trial type: $\mathrm{F}(4,33)=0.66, p=0.30)$. PPF in young men did not differ from that in postmenopausal women $(\mathrm{F}(1,35)=0.321, p=0.57)$ at any interval (group $\times$ trial type: $\mathrm{F}(4,32)=0.42, p=0.79$ ).

Subsample analyses: There was only a significant main effect of trial type $(\mathrm{F}(4,54)=11.49, p<0.001)$ and trends for the main effect of age group $(\mathrm{F}(1,57)=3.67, p=0.06)$ and a sex $\times$ age group interaction $(\mathrm{F}(1,57)=2.28, p=0.13)$ (mean \pm 1 SEM PPF with 1000, 2000, 3000, 4500, and $6000 \mathrm{~ms}$ trials, respectively, in premenopausal women: $-0.83 \pm 5.21,-2.775 \pm 5.03,-8.91 \pm 4.93,-15.40 \pm 5.40$, and $-9.17 \pm 4.57$; young men: $1.04 \pm 5.74,-3.8 \pm 5.54,-13.20 \pm$ $5.43,-18.91 \pm 5.95$, and $-11.68 \pm 5.03$; postmenopausal women: $2.83 \pm 5.37,-2.63 \pm 5.18,-8.58 \pm 5.08,-14.59 \pm 5.57$, and $-6.30 \pm 4.71$; older men: $8.59 \pm 5.74,4.77 \pm 5.54,6.72 \pm$ $5.43,-3.87 \pm 5.90$, and $3.04 \pm 5.03)$.
Premenopausal women and young men did not differ in PPF (sex: $F(1,29)=0.11, p=0.74)$. There was a trend for postmenopausal women to show greater PPF than older men $(\mathrm{F}(1,28)=3.62, p=0.07)$.

Pre- and postmenopausal women showed comparable PPF (age group: $F(1,31)=0.09, p=0.77$ ). Reflecting the finding noted above, young men showed greater PPF than older men (age group: $\mathrm{F}(1,26)=5.70, p=0.02$ ).

Further between-group analyses revealed that premenopausal women showed greater PPF than older men (group: $\mathrm{F}(1,29)=7.82, p=0.009)$. PPF in young men did not differ from that in postmenopausal women $(\mathrm{F}(1,28)=0.27, p=0.60)$.

Response amplitude and habituation. There was no difference between men and women in amplitude or habituation rate over three blocks of pulse-alone trials (block, $\mathrm{F}(2,70)=9.30, p<0.001$; linear, $\mathrm{F}(1,71)=14.66$, $p<0.001$; sex, $\mathrm{F}(1,71)=0.13, p=0.72$; sex $\times$ block interaction, $\mathrm{F}(2,70)=1.08, p=0.34)$. There was no difference between young and older participants in habituation rate (age group $\times$ block interaction, $\mathrm{F}(2,70)=0.18, p=0.84$ ), but older participants, regardless of sex, had higher amplitude on all three blocks compared to young participants (age group, $\mathrm{F}(1,71)=8.76, p=0.004$ ) (Table 1). Exclusion of eight older participants who were taking blood pressure/ cholesterol-lowering medication did not abolish the effect of age group in pulse-alone amplitude $(\mathrm{F}(1,63)=4.82$, $p=0.03$ ); other effects also remained unchanged.

Although mean pulse-alone amplitude was higher in older participants, it did not correlate positively or negatively with PPI or PPF at any interval in the entire sample or when examined separately in two sexes or the two age groups, except correlating positively with $4500 \mathrm{~ms}$ PPF in young participants $(r=0.40, p=0.01)$. Given that a large number $(>50)$ of correlations were performed, this one correlation is likely to be a spurious finding.

Sex or age group had no effect on their own or in interaction in initial reactivity, as measured by amplitude on the first pulse-alone trial (all $p>0.10)$.

\section{Latencies to response peak.}

PPI: There was a main effect of trial type $(\mathrm{F}(5,67)=3.61$, $p=0.006)$ showing significant latency facilitation by $30 \mathrm{~ms}$ $(t(74)=4.15, \quad p<0.001)$ and $60 \mathrm{~ms}$ prepulse trials $(t(74)=2.34, p=0.02)$, relative to pulse-alone trials; latencies for other trials did not show significant facilitation or inhibition (all $p>0.10$; see Table 1 mean values). There was a marginally significant sex $\times$ age group interaction $(\mathrm{F}(1,71)=3.89, p=0.05)$, indicating faster latencies in premenopausal women than young men $(\mathrm{F}(1,35)=4.75$, $p=0.04$ ) but no difference between postmenopausal women and older men $(\mathrm{F}(1,36)=0.48, p=0.50)$. The effect of age group was not significant in men or women (all $p>0.10$ ).

PPF: There was a trend for the main effect of trial type $(\mathrm{F}(5,67)=2.05, p=0.08)$ and a significant sex $\times$ trial type interaction $(\mathrm{F}(5,67)=2.56, p=0.04)$. Further analysis revealed a main effect of trial type $(\mathrm{F}(5,30)=4.07$, $p=0.006$; indicating latency facilitation by $1000 \mathrm{~ms}$ PPF trials, $t(34)=2.12, p=0.04)$ in men but not in women $(\mathrm{F}(5,35)=1.68, p=0.16)$. 
Table I Mean (SEM) Amplitudes (in Analogue-To-Digit Units; I Unit = $2.62 \mathrm{mV}$ ) over Three Blocks of Pulse-Alone Trials, and Latencies to Response Peak (ms) for Pulse-Alone Trials and Prepulse Inhibition/Facilitation Trials for the Four Study Groups

\begin{tabular}{|c|c|c|c|c|}
\hline \multirow[b]{2}{*}{ Measure } & \multicolumn{2}{|c|}{ Aged $20-40$ years } & \multicolumn{2}{|c|}{ Aged 55-69 years } \\
\hline & Women $(n=20)$ & Men $(n=17)$ & Women $(n=20)$ & Men $(n=18)$ \\
\hline Block I & $425.11(71.01)$ & $339.08(53.37)$ & $543.95(85.46)$ & $658.18(100.87)$ \\
\hline Block 2 & $304.78(57.63)$ & $250.07(38.55)$ & $498.7 \mid(107.40)$ & $508.65(87.98)$ \\
\hline \multicolumn{5}{|c|}{ Latencies to response peak for pulse-alone, PPI, and PPF trials } \\
\hline Pulse-alone & $59.05(9.07)$ & $65.34(1.57)$ & $65.02(2.00)$ & $60.48(2.68)$ \\
\hline \multicolumn{5}{|l|}{ PPI } \\
\hline 480-ms prepulse-to-pulse interval & $60.20(2.93)$ & $62.66(1.16)$ & $60.34(2.58)$ & $60.33(2.59)$ \\
\hline \multicolumn{5}{|l|}{ PPF } \\
\hline I000-ms prepulse-to-pulse interval & $58.66(2.32)$ & $63.40(1.99)$ & $62.49(2.50)$ & $57.56(2.56)$ \\
\hline 2000-ms prepulse-to-pulse interval & $58.89(2.29)$ & $62.48(1.05)$ & $64.20(2.44)$ & $57.31(3.02)$ \\
\hline 3000-ms prepulse-to-pulse interval & $58.60(1.99)$ & $62.71(1.29)$ & $59.03(2.38)$ & $60.59(2.27)$ \\
\hline 4500-ms prepulse-to-pulse interval & $60.85(2.23)$ & $65.59(1.23)$ & $59.78(2.7 \mid)$ & $62.19(2.22)$ \\
\hline 6000-ms prepulse-to-pulse interval & $61.11(1.91)$ & $63.04(1.29)$ & $63.17(2.62)$ & $59.98(3.18)$ \\
\hline
\end{tabular}

Sex hormones. Hormones data were unavailable for 4 (3 men ( 2 young and 1 aged) and 1 premenopausal woman) of 75 participants due to technical failures or other reasons.

$17 \beta$-Estradiol: There was a main effect of age group $(\mathrm{F}(1,71)=12.56, p=0.001)$, indicating a higher level, on average, in young compared with aged participants, and a sex $\times$ age group interaction $(\mathrm{F}(1,71)=3.96, p=0.05)$, indicating a much higher level in pre- compared with postmenopausal women $(t(37)=3.91, p<0.001)$ but no difference between the young and aged men $(t(30)=1.13$, $p=0.27$ ) (mean $\pm 1 \mathrm{SD}$ values $(\mathrm{pg} / \mathrm{ml})$, premenopausal woman: $6.03 \pm 4.61$; young men: $4.33 \pm 3.65$; postmenopausal women: $2.17 \pm 1.46$; and older men: $3.10 \pm 1.83$ ).

Testosterone: There was only a significant main effect of $\operatorname{sex}(\mathrm{F}(1,71)=5.41, p=0.02)$, indicating a higher level in men, relative to women (mean $\pm 1 \mathrm{SD}$ values $(\mathrm{pg} / \mathrm{ml}$ ), premenopausal woman: $44.36 \pm 39.83$; young men: $71.09 \pm$ 50.72 ; postmenopausal women: $23.59 \pm 27.08$; and older men: $68.72 \pm 26.75)$.

PPI/PPF and sex hormones. The results of correlational analyses (Table 2 ) revealed that $120 \mathrm{~ms}$ PPI did not correlate with the levels of $17 \beta$-estradiol or testosterone. Greater $4500 \mathrm{~ms}$ PPF (scored as greater negative value) correlated with higher $17 \beta$-estradiol and higher testosterone levels. The significant association was present mainly in young men (Table 2).

\section{DISCUSSION}

The findings confirmed our hypothesis of less PPI in premenopausal, but not postmenopausal, women compared with men of similar ages. In women, there was no significant difference in PPI between pre- and postmenopausal groups. In men, however, the young group showed more PPI than the older group. Our findings mirror closely to those of a recent study in CBA/CaJ mice by Ison and Allen (2007), who observed (i) less PPI in premenopausal (aged 3-8 months) females compared with similarly aged males, (ii) no difference in PPI between postmenopausal (aged 17-25 months) females and similarly aged males, (iii) less PPI in 17-25 months old males, relative to 3-8 months old males, and (iv) no difference in PPI between pre- and postmenopausal mice. There can be more than one explanation for the pattern of results obtained in our study as proposed earlier by Ison and Allen (2007) for their findings in mice.

The first explanation, in line with previous data in humans (Swerdlow et al, 1997; Jovanovic et al, 2004) as well as rats (Koch, 1998), is that complex hormonal fluctuations over the menstrual cycle are responsible for less PPI in premenopausal young women compared with young men, and no difference in PPI between postmenopausal women and older men. However, the loss of sex difference in PPI when examined in postmenopausal women and older men was due mainly to lower PPI in the aged, relative to young, men. This fits better with a different explanation that the PPI advantage in men also includes an influence of male sex 
Table 2 Correlations between PPI/PPF Levels and Sex Hormones

\begin{tabular}{|c|c|c|c|c|}
\hline Measure & Group & PPF 4500-ms & |7 $\beta$-Estradiol & Testosterone \\
\hline \multirow[t]{8}{*}{ |20-ms PPI } & All & 0.054 & 0.164 & 0.175 \\
\hline & Women (all) & 0.112 & 0.131 & 0.19 \\
\hline & Men (all) & -0.062 & 0.294 & -0.097 \\
\hline & Young (all) & -0.036 & -0.056 & 0.183 \\
\hline & Premenopausal women & 0.027 & -0.102 & 0.202 \\
\hline & Young men & 0.125 & 0.258 & -0.059 \\
\hline & Postmenopausal women & 0.182 & 0.21 & 0.092 \\
\hline & Older men & 0.311 & 0.066 & -0.052 \\
\hline \multirow{7}{*}{ 4500-ms PPF } & Men (all) & - & $-0.448 * *(0.010)$ & $-0.502 * * *(0.003)$ \\
\hline & Young (all) & - & -0.302 & $-0.379 *(0.027)$ \\
\hline & Older (all) & - & -0.028 & 0.072 \\
\hline & Premenopausal women & - & -0.247 & -0.083 \\
\hline & Young men & - & $-0.456^{\#}$ & $-0.558 *(0.03 I)$ \\
\hline & Postmenopausal women & - & -0.056 & -0.02 \\
\hline & Older men & - & -0.248 & $-0.37 \mid$ \\
\hline
\end{tabular}

${ }^{*} p=0.05 ;{ }^{* *} p=0.01 ;{ }^{*} * * * 0.005 ;{ }^{*} p=0.09$. Negative relationship between PPF and hormones means indicates that more PPF is associated with higher hormone levels.

hormones, such as testosterone, that decline with age (Freeman et al, 2001). The data from two previous human studies (Ludewig et al, 2003; Jovanovic et al, 2004) showing no difference in PPI between men and women tested during the follicular phase argue against this explanation. However, none of these two studies obtained hormonal measurements, and both had 15 or fewer participants available for follicular phase women $v s$ men comparison. Other studies (eg, Swerdlow et al, 1997; Abel et al, 1998; Kumari et al, 2003) have shown less PPI in women even when tested during the follicular phase relative to men. These data combine to suggest that although women show the greatest level of PPI during the follicular phase, there may still be some (young) male PPI advantage due to male sex hormones, in particular testosterone. Further support for a role of testosterone in male PPI comes from the observation that the effect of serotonin-1a receptor stimulation on PPI in male rats depends more strongly on circulating levels of testosterone than estrogen (Gogos and van den Buuse, 2003). The effect of age in PPI was much less pronounced and nonsignificant in women, perhaps off-set by the absence of strong cyclic hormonal fluctuations.

The age effect in PPI we found in men is consistent with the observations of reduced PPI in old rats (Varty et al, 1998). The results of previous studies of age effects in human PPI have been inconsistent. Ellwanger et al (2003) examined PPI in four (mixed-sex) age groups: college students (18-24 years), young subjects (25-34 years), middle-aged subjects (36-50 years), and old subjects (5988 years). They observed a curvilinear relationship between age and PPI. More specifically, they found greatest percentage of PPI in the middle-aged group and the lowest percentage of PPI in the college students group and, although the old group (59-88 years) did not differ significantly from other groups, there was less PPI in the old group relative to the middle-aged group. The design of our study is not directly comparable to their design as our young group overlaps with three of their groups (college students, young, and middle-aged). The influence of sex in PPI-reducing effect of age, combined with nonlinear relationship between age and PPI, may at least, in part, explain previously reported negative findings on age effect in human PPI (eg, Ludewig et al, 2003). Another related issue deserving some discussion is that there was generally less PPI in young as well as aged subjects in our study (prepulse intensity $14 \mathrm{~dB}$ above background) than in the study of Ellwanger et al (prepulse intensity $16 \mathrm{~dB}$ above background). It is possible that, in addition to prepulse intensity, subject characteristics in their and our studies varied on important dimensions, for example cigarette smoking, that are known to influence PPI (review, Braff et al, 2001). In our study, only 5 (2 young and 1 aged men, and 2 postmenopausal women) of 75 subjects smoked (5-12 cigarettes/day), and they too did not smoke prior to testing. The smoking status for participants in Ellwanger's study is not reported. Another study by Hejl et al (2004) showed 
considerable PPI ( $>60 \%$ with 120 -ms prepulse-to-pulse interval; prepulse intensity $15 \mathrm{~dB}$ above background) in an aged mixed-sex group of healthy controls $(n=49$; mean age 70.2 years; 19/49 considered startle nonresponders and excluded). This study, however, included subjects on a range of medications, including estrogen substitution therapy, making direct comparison of data from our and their studies difficult. More recently, Ueki et al (2006) reported PPI level (prepulse intensity $15 \mathrm{~dB}$ above background) in an aged mixed-sex healthy control group ( $n=30,60 \%$ men, mean age 66.7 years; none on any medication, smoking status not reported) similar to that seen in our aged groups. PPI is known to be sensitive to stimulus parameters, test conditions, and subject characteristics with substantial variability in PPI distribution in normal healthy populations across studies and laboratories (review, Braff et al, 2001). It is possible that inhibition generated by prepulses under different test and stimulus conditions is not mediated by exactly the same physiological substrates and shows differential sensitivity to the effects of sex, age, and hormones.

We, contrary to our expectation, observed a sex difference in PPF indicating more PPF in postmenopausal, but not in premenopausal, women relative to similarly aged men. Preand postmenopausal groups of women showed comparable PPF and only young men showed PPF comparable to that seen in women; older men did not show PPF at all (Figure 1). This pattern of results suggests that PPF is influenced by age in men and, therefore, may be sensitive to male sex hormones, which decline with age. The positive association found between the levels of PPF and testosterone in men further supports such as association. The finding of comparable PPF in young premenopausal women and young men fails to support our earlier proposition (Kumari et al, 2003) that sex differences in human sensorimotor gating might represent a general downshift in the inhibition curve and upward shift in the facilitation curve in women compared with men. However, higher $17 \beta$-estradiol level also related to greater PPF in this study. This combined with high estrogen-low PPI association (Swerdlow et al, 1997; Jovanovic et al, 2004) may give rise to such a pattern of sex differences if both associations are present at the same time.

Despite observing expected sex differences in PPI when premenopusal women were compared with young men, we did not find any association between individual differences in the endogenous levels of sex hormones and PPI. We had expected a negative relationship between PPI and $17 \beta$ estradiol level in young women. Previous studies utilizing both between- (Swerdlow et al, 1997) and within-subjects designs (Jovanovic et al, 2004) have shown reduced PPI in the luteal, relative to the follicular, phase in healthy young women, and this effect has been attributed to elevated estrogen levels during the luteal phase (Koch, 1998). It is perhaps worth pointing out that although we did not find a significant negative relationship between PPI and $17 \beta$-estradiol levels in young premenopausal women, the direction of this association for this particular group, unlike the other three groups, was negative (Table 2). As has been proposed to be the case for testosterone and spatial cognition (Kimura, 1999), there may exist a nonlinear relationship between PPI and estrogen levels with both very low and very high levels associating with lower PPI. However, when tested formally, neither quadratic nor linear relationship was significant in the current data set $(p>0.15)$. A number of previous studies have also failed to find a relationship between individual differences in the endogenous levels of estrogen and performance on sexually dimorphic cognitive functions (eg, Gordon and Lee, 1993; Halari et al, 2005). Within-subject hormonal changes as a function of natural fluctuations over the ovarian cycle or produced by hormone administration in deficient populations may have stronger associations with PPI than individual differences in endogenous levels of circulating sex hormones.

An unexpected finding of this study concerns higher startle amplitudes in older, relative to young, participants. This is opposite to what would normally be expected (Ludewig et al, 2003). It is possible that our older participants were aroused during the testing procedure. The majority of them were recruited specifically for this project and had no prior experience of taking part in psychophysiological research. Young participants, on the other hand, were recruited from an established departmental database and very likely to have taken part in multiple psychological studies and had been exposed to psychophysiological laboratory environment. The sex effect seen in PPI and PPF, however, was unrelated to observed age effect in amplitude.

In conclusion, the main finding of this study demonstrates reduced PPI in pre-, but not postmenopausal, women relative to men of similar ages and extends recent observations (Ison and Allen, 2007) in mice showing less PPI in pre- but not postmenopausal female compared to male mice of similar ages to humans. Other findings of this study suggest that PPI and PPF may be sensitive to male sex hormones, in addition to female sex hormones. However, there appear to be no substantial relationships between individual differences in endogenous levels of estrogen and PPI; fluctuations within an individual may play a stronger role. Further within-subject studies examining the influence of natural hormonal fluctuations in women and hormone administration to deficient populations with concurrent measurements of not only the levels of estrogen but also of progesterone and testosterone are required. Future studies should also consider the length of the menopause in postmenopausal women, as the duration of hypogonadism may affect neural systems modulated by sex steroids.

\section{ACKNOWLEDGEMENTS}

This study was funded by a grant (SG-39919 to VK and AC) from the British Academy (UK). We are grateful to Mrs Irene Papadopoulos for her help with the study.

\section{DISCLOSURE/CONFLICT OF INTEREST}

The authors declare no conflict of interest.

\section{REFERENCES}

Aasen A, Kolli L, Kumari V (2005). Sex effects in prepulse inhibition and facilitation of the acoustic startle response: implications for pharmacological and treatment studies. J Psychopharmacol 19: 39-45. 
Abel K, Waikar M, Pedro B, Hemsley D, Geyer M (1998). Repeated testing of prepulse inhibition and habituation of the startle reflex: a study in healthy human controls. J Psychopharmacol 12: 330-337.

Anthony BJ (1985). In the blink of an eye: implications of reflex modification for information processing. In: Ackles PK, Jenings JR, Coles MGH (eds). Advances in Psychophysiology Vol 1, JAI Press: Greenwich, CT, 167-218.

Braff DL, Geyer MA (1990). Sensorimotor gating and schizophrenia. Arch Gen Psychiatry 47: 181-188.

Braff DL, Geyer MA, Swerdlow NR (2001). Human studies of prepulse inhibition of startle: normal subjects, patient groups, and pharmacological studies. Psychopharmacology 156: 234-258.

Dawson ME, Schell AM, Swerdlow NE, Filion DL (1997). Cognitive, clinical, and neuropsychological implications of startle modulation. In: Lang PJ, Simons RF, Balaban M (eds). Attention and Orienting: Sensory and Motivational Processes. Lawrence Erlbaum Associates: Mahwah, NJ, 257-279.

Ellwanger J, Geyer MA, Braff DL (2003). The relationship of age to prepulse inhibition and habituation of the acoustic startle response. Biol Psychol 62: 175-195.

Faraday MM, O’Donoghue VA, Grunberg NE (1999). Effects of nicotine and stress on startle amplitude and sensory gating depend on rat strain and sex. Pharmacol Biochem Behav 62: 273-284.

Freeman ER, Bloom DA, McGuire EJ (2001). A brief history of testosterone. J Urol 165: 371-373.

Gogos A, van den Buuse M (2003). Castration reduces the effect of serotonin-1A receptor stimulation on prepulse inhibition in rats. Behav Neurosci 117: 1407-1415.

Gordon HW, Lee PL (1993). No differences in cognitive performance between phases of the menstrual cycle. Psychoneuroendocrinology 18: 521-531.

Graham FK (1975). The more or less startling effects of weak prestimuli. Psychophysiology 12: 238-248.

Graham FK, Murray GM (1977). Discordant effects of weak prestimulation on magnitude and latency of the blink reflex. Physiol Psychol 5: 108-114.

Halari R, Hines M, Kumari V, Mehrotra R, Wheeler M, Ng V et al. (2005). Sex differences and individual differences in cognitive performance and their relationship to endogenous gonadal hormones and gonadotropins. Behav Neurosci 119: 104-117.

Halbreich U (1995). Menstrually related disorders: what we do know, what we only believe that we know, and what we know that we do not know. Cr Rev Neurobiol 9: 163-175.

Hazlett EA, Buchsbaum MS, Haznedar MM, Singer MB, Germans MK, Schnur DB et al (1998). Prefrontal cortex glucose metabolism and startle eyeblink modification abnormalities in unmedicated schizophrenia patients. Psychophysiology 35: 186-198.
Hejl AM, Glenth $\varnothing \mathbf{j}$ B, Mackeprang T, Hemmingsen R, Waldemar G (2004). Prepulse inhibition in patients with Alzheimer's disease. Neurobiol Aging 25: 1045-1050.

Hoffman HS, Searle JL (1968). Acoustic and temporal factors in the evocation of startle. J Acoust Soc Am 43: 269-282.

Ison JR, Allen PD (2007). Pre- but not post-menopausal female $\mathrm{CBA} / \mathrm{CaJ}$ mice show less prepulse inhibition than male mice of the same age. Behav Brain Res 185: 76-81.

Jovanovic T, Szilagyi S, Chakravorty S, Fiallos AM, Lewison BJ, Parwani A et al (2004). Menstrual cycle phase effects on prepulse inhibition of acoustic startle. Psychophysiology 41: 401-406.

Kimura D (1999). Sex and Cognition. A Bradford Book, MIT Press: Cambridge, MA.

Koch M (1998). Sensorimotor gating changes across the estrous cycle in female rats. Physiol Behav 64: 625-628.

Kumari V, Aasen I, Sharma T (2004). Sex effects in prepulse modification deficits in schizophrenia. Schizophr Res 69: 219-235.

Kumari V, Gray J, Gupta P, Luscher S, Sharma T (2003). Sex differences in prepulse inhibition of the acoustic startle response. Pers Indiv Diff 34: 733-742.

Landis C, Hunt WA (1939). The Startle Pattern. Farrar \& Rinehart: New York.

Ludewig K, Ludewig S, Seitz A, Obrist M, Geyer MA, Vollenweider FX (2003). Acoustic startle reflex and its modulation: effects of age and gender in humans. Biol Psychol 63: 311-323.

Mansbach RS, Geyer MA, Braff DL (1988). Dopaminergic stimulation disrupts sensorimotor gating in the rat. Psychopharmacology 94: 507-514.

Park SJ, Goldsmith LT, Weiss G (2002). Age-related changes in the regulation of luteinizing hormone secretion by estrogen in women. Exp Biol Med 227: 455-464.

Swerdlow NR, Auerbach A, Monroe SM, Hartson H, Geyer MA, Braff DL (1993). Men are more inhibited than women by weak prepulses. Biol Psychiatry 34: 253-260.

Swerdlow NR, Geyer MA, Hartman PL, Sprock J, Auerbach PP, Cadenhead K (1999). Sex differences in sensorimotor gating of the human startle response: all smoke? Psychopharmacology 146: 228-232.

Swerdlow NR, Hartson H, Auerbach A (1997). Changes in sensorimotor inhibition across the menstrual cycle: implications for neuropsychiatric disorders. Biol Psychiatry 41: 452-460.

Ueki A, Goto K, Sato N, Iso H, Morita Y (2006). Prepulse inhibition of acoustic startle response in mild cognitive impairment and mild dementia of Alzheimer type. Psychiatry Clin Neurosci 60: $55-62$.

Varty GB, Hauger RL, Geyer MA (1998). Aging effects on the startle response and startle plasticity in Fischer F344 rats. Neurobiol Aging 19: 243-251. 